\title{
Magnetic irreversibility: An important amendment in the zero-field-cooling and field-cooling method
}

Fábio Teixeira Dias1*, Valdemar das Neves Vieira1, Sabrina Esperança Nunes1, Paulo Pureur2, Jacob Schaf2,

Graziele Fernanda Farinela da Silva2, Cristol de Paiva Gouvêa3, Frederik Wolff-Fabris4, Erik Kampert4,

Xavier Obradors5, Teresa Puig5, and Joan Josep Roa Rovira6

1 Instituto de Física e Matemática, Universidade Federal de Pelotas, C.P. 354, 96010-900

Pelotas, Rio Grande do Sul, Brazil

2Instituto de Física, Universidade Federal do Rio Grande do Sul, 91501-970 Porto Alegre, Rio Grande do Sul, Brazil

3Divisão de Metrologia de Materiais, Inmetro, 25250-020 Duque de Caxias, Rio de Janeiro, Brazil

4Dresden High Magnetic Field Laboratory, Helmholtz-Zentrum Dresden-Rossendorf, 01314 Dresden, Germany

5Institut de Ciència de Materials de Barcelona, C.S.I.C., Campus Universitat Autònoma de Barcelona, 08193 Bellaterra, Spain

6Departamento de Ciencia de Materiales e Ingeniería Metalúrgica, Universitat Politècnica de Catalunya, 08028 Barcelona, Spain

\section{Abstract}

The present work reports about experimental procedures to correct significant deviations of magnetization data, caused by magnetic relaxation, due to small field cycling by sample transport in the inhomogeneous applied magnetic field of commercial magnetometers. The extensively used method for measuring the magnetic irreversibility by first cooling the sample in zero field, switching on a constant applied magnetic field and measuring the magnetization $M(T)$ while slowly warming the sample, and subsequently measuring $M(T)$ while slowly cooling it back in the same field, is very sensitive even to small displacement of the magnetization curve. In our melt-processed $\mathrm{YBaCuO}$ superconducting sample we observed displacements of the irreversibility limit up to $7 \mathrm{~K}$ in high fields. Such displacements are detected only on 
confronting the magnetic irreversibility limit with other measurements, like for instance zero resistance, in which the sample remains fixed and so is not affected by such relaxation. We measured the magnetic irreversibility, $\operatorname{Tirr}(\mathrm{H})$, using a vibrating sample magnetometer (VSM) from Quantum Design. The zero resistance data, $\mathrm{TcO}(\mathrm{H})$, were obtained using a PPMS from Quantum Design. On confronting our irreversibility lines with those of zero resistance, we observed that the $\mathrm{TCO}(\mathrm{H})$ data fell several degrees $\mathrm{K}$ above the $\operatorname{Tirr}(\mathrm{H})$ data, which obviously contradicts the well known properties of superconductivity. In order to get consistent $\operatorname{Tirr}(\mathrm{H})$ data in the $\mathrm{H}-\mathrm{T}$ plane, it was necessary to do a lot of additional measurements as a function of the amplitude of the sample transport and extrapolate the $\operatorname{Tirr}(\mathrm{H})$ data for each applied field to zero amplitude.

\section{Introduction}

In homogeneous superconductors a non-zero electric current can flow without any resistance below the superconducting transition temperature (Tc). However, if a magnetic field is present within the superconductor, the electric current may interact with the quantized magnetic fluxons, causing magnetic flux dynamics and thereby dissipating energy resulting in resistivity. If the superconductor contains a large amount of strong flux pinning centers, flux pinning may suppress the flux dynamics, allowing a non-zero electric current to flow without dissipation. In reality, however, closely underneath to the superconducting transition temperature the pinning is too weak to suppress flux dynamics. It succeeds only at a certain range below Tc, along a boundary in the field-temperature $(\mathrm{H}-\mathrm{T})$ plane, known as the magnetic irreversibility line, $\operatorname{Tir}(\mathrm{H})$. Theoretically below $\operatorname{Tirr}(\mathrm{H})$ a nonzero electric current may flow without resistance. On the other hand, it is absolutely impossible to a current to flow without resistance above the irreversibility line. Moreover, in non-homogeneous or granular superconductors, while low magnetic irreversibility begins already when the first grain clusters couple together, stabilizing Josephson fluxons, the electric resistance vanishes only when the superconducting order parameter of the grains percolates through the whole sample.1-4)

\section{Sample preparation}

Our melt-processed YBa2Cu3O7- $\delta$ (Y123) sample, doped with $30 \%$ of non-superconducting Y2BaCuO5 (Y211) particles, was produced by the Institute of Materials Science of Barcelona and at the Department of Materials Science and Metallurgical Engineering of the University of Barcelona, in Spain. More information about the sample preparation and treatment may be found in Refs. 5-12. The non-superconducting Y211 particles provide the Y123 sample with a high density of very effective flux pinning centers.13) The high quality melt-processed Y123 
sample has highly aligned crystallite c-axis and a very strong superconducting linking between the crystallites along the ab plane. In spite of their polycrystalline structure, the high quality of the melt-processed materials is well known to do not exhibit the signature of superconducting granularity. This granularity is characterized by the de Almeida-Thouless14) and the GabayToulouse15) like functionality in the low field magnetic irreversibility line.

The melt-processed Y123 sample, used in the present study, was a polycrystalline parallelepiped with $4 \times 1.8 \mathrm{~mm} 2$ in the ab plane and $2 \mathrm{~mm}$ along the c-axis. It was characterized by scanning electron microscopy in order to verify the grain alignment. Figure 1 is a scanning electron microscope (SEM) image, obtained with the Fei Nova Nanolab 600. The image shows the profiles of long superconducting platelets along the ab plane stacked along the c-axis.

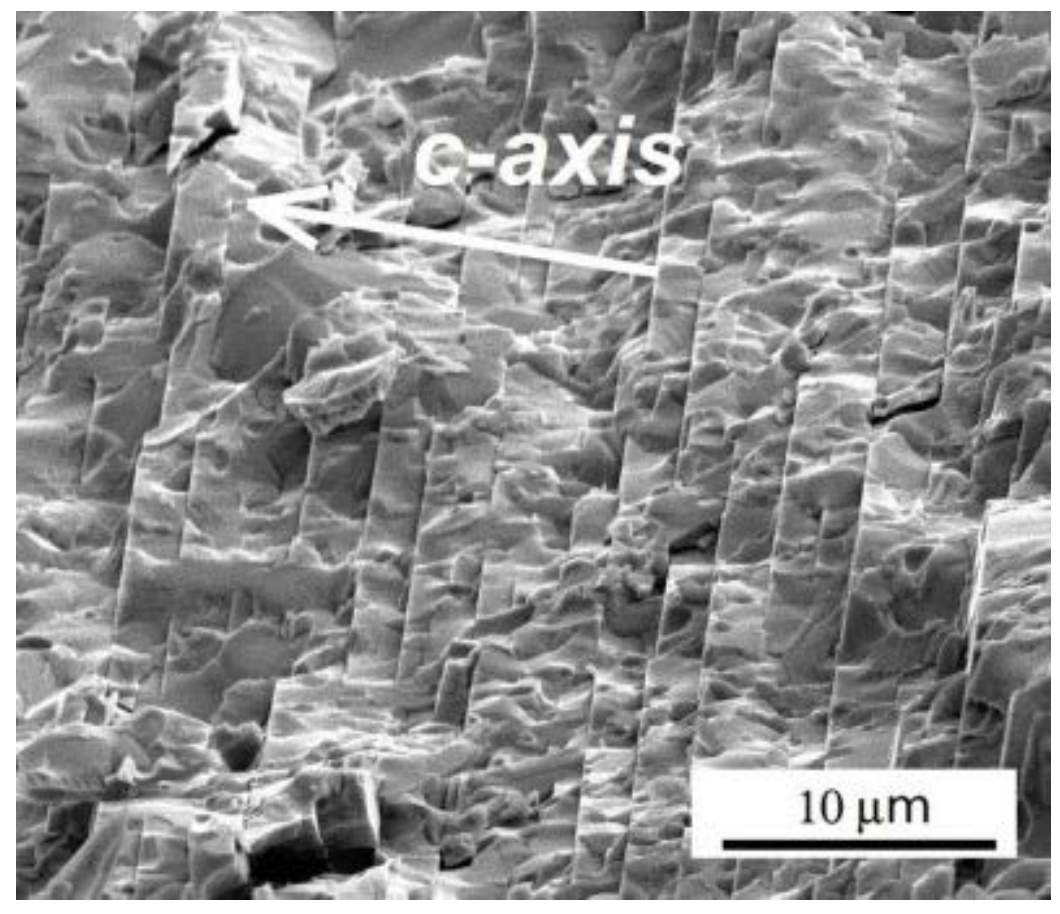

Fig. 1. (Color online) SEM image of the Y123 sample, showing profiles of the platelet structure stacked along the c-axis because of the texture process.

\section{Experimental procedure}

The electric resistance measurements were made as a function of temperature using a physical properties measurement system (PPMS) from Quantum Design under constant applied magnetic fields ranging from zero up to $90 \mathrm{kOe}$. Four good electrical contacts on the samples were achieved by heating them with freshly painted silver paint stripes to $400{ }^{\circ} \mathrm{C}$ in pure oxygen for $1 \mathrm{~h}$. The magnetoresistance experiments were performed by applying a low measuring current density parallel to the ab plane and along the c-axis in five different field- 
current configurations, namely $\mathrm{H}\|\mathrm{J}\| \mathrm{ab}, \mathrm{H}\|\mathrm{ab}-\mathrm{J}\| \mathrm{ab}(\mathrm{H} \perp \mathrm{J}), \mathrm{H} \|$ ab-J \| c, H \| c-J \| c, H \| c-J \| ab. We obtained a high density of experimental data while slowly cooling the samples through the superconducting transition region under constant applied magnetic fields. The sample used in the magnetoresistance measurements was the same used in the magnetic measurements.

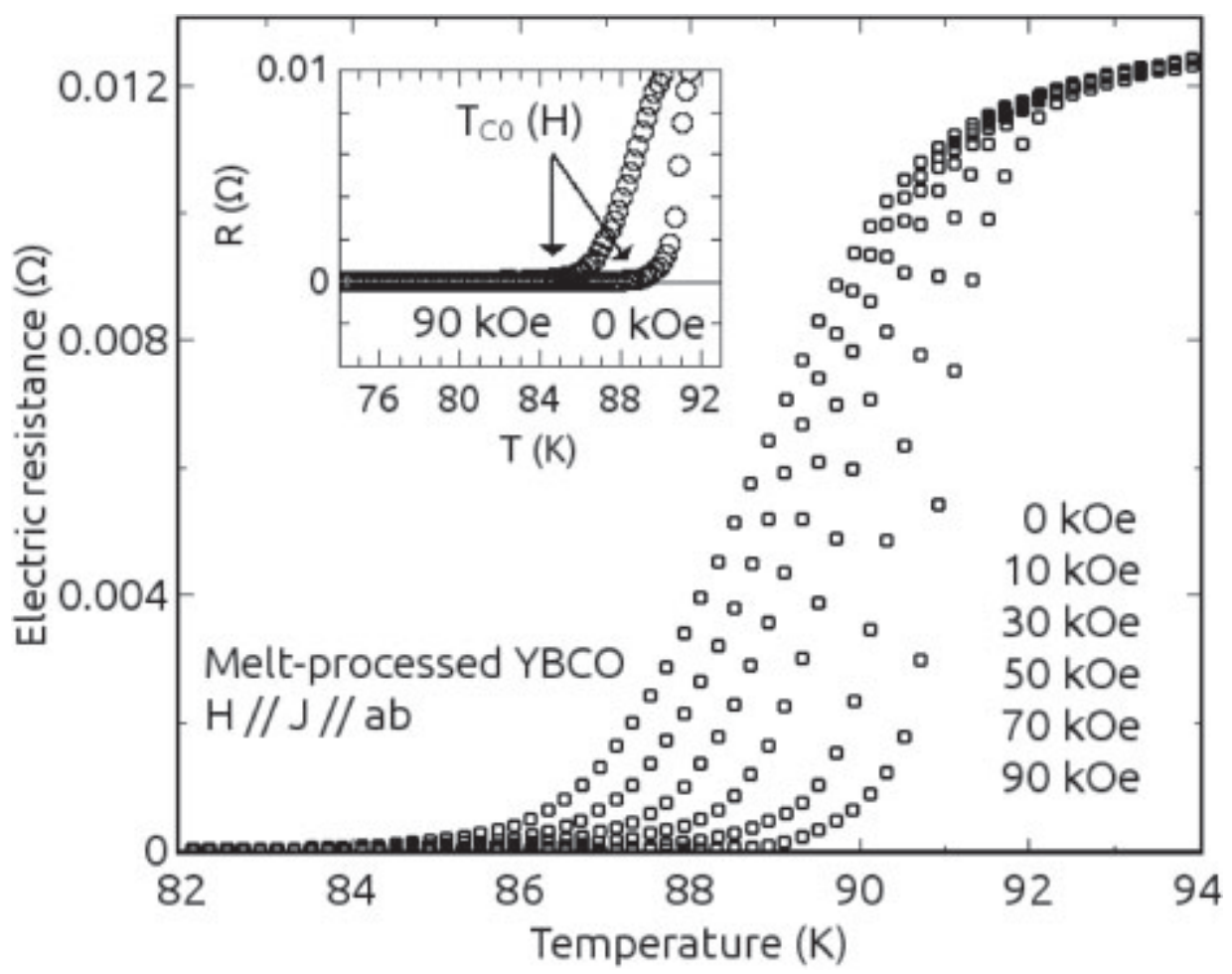

Fig. 2. Electric resistance data of the sample in the configuration $\mathrm{H}\|\mathrm{J}\|$ ab from 0 up to 90 kOe. The inset highlights the zero resistance temperature point for the magnetic fields of 0 and $90 \mathrm{kOe}$, denoted as $\mathrm{TCO}(\mathrm{H})$.

Figure 2 displays representative magnetoresistance data of our doped melt-processed Y123 sample for several magnetic fields within the range from 0 up to $90 \mathrm{kOe}$, applied parallel to the electric current and both along the ab plane. The purpose of this figure is illustrating our experimental method and data analysis. In our data analysis the zero resistance temperature, denoted by $\mathrm{TcO}$, is obtained by a practical criterion, according to which the zero resistance temperature is the threshold of the plateau where $d R=d T$ falls to zero. Usually, the effective zero depends on many details of the sample installation. Therefore, the generally used criterion for zero resistance is the appearance of an effectively flat plateau. This is reliable, because non-zero resistance certainly would show up a slope as a function of temperature. The inset of Fig. 2 shows details of the method used to find the limit of zero resistance. The 
arrows in the inset indicate the point where the electric resistance becomes zero according to our criterion, for 0 and $90 \mathrm{kOe}$.

The magnetization measurements were made on the same sample used in the electric resistance measurements, using a vibrating sample magnetometer (VSM) from Quantum Design, in magnetic fields from 0.01 to $50 \mathrm{kOe}$ applied along the c-axis or parallel to the ab plane. The magnetic irreversibility limit was obtained by using the standard method, based on a zero-field-cooling (ZFC) process followed by a field-cooling (FC) process. The ZFC consists in cooling the sample first down to a desired temperature in zero applied field, then switching on a chosen field and subsequently measuring the magnetization $M(T)$ while slowly warming the sample ( $\sim 0.2 \mathrm{~K}=\mathrm{min}$ or less) in the constant field (persistent mode) up to a temperature above the transition temperature. The FC consists in measuring $M(T)$ while slowly cooling the sample back in the same constant field.

In order to obtain a meaningful comparison between magnetic irreversibility and transport data, the temperature sensors and magnets should be precisely calibrated with respect to each other. Our data were corrected for temperature gradient effects, which always were less than $0.2 \mathrm{~K}$. The irreversibility limit $\operatorname{Tirr}(\mathrm{H})$ for a given applied field is the temperature where the difference between FC and ZFC magnetic long moments deviates definitely from the zero baseline defined by the high-temperature data, where the magnetic moment is reversible. Figure 3 illustrates our criterion for finding the magnetic irreversibility limit. The main figure represents the difference between FC and ZFC magnetic moments for the melt-processed sample under a magnetic field of $50 \mathrm{kOe}$, applied along the ab plane and for two different amplitudes of the sample transport into the magnetometer, namely, 1 and $5 \mathrm{~mm}$. The arrows indicate the irreversibility limit Tirr(H) for the chosen field and the inset shows the original ZFC and FC curves, in this case for a sample transport of $5 \mathrm{~mm}$. 


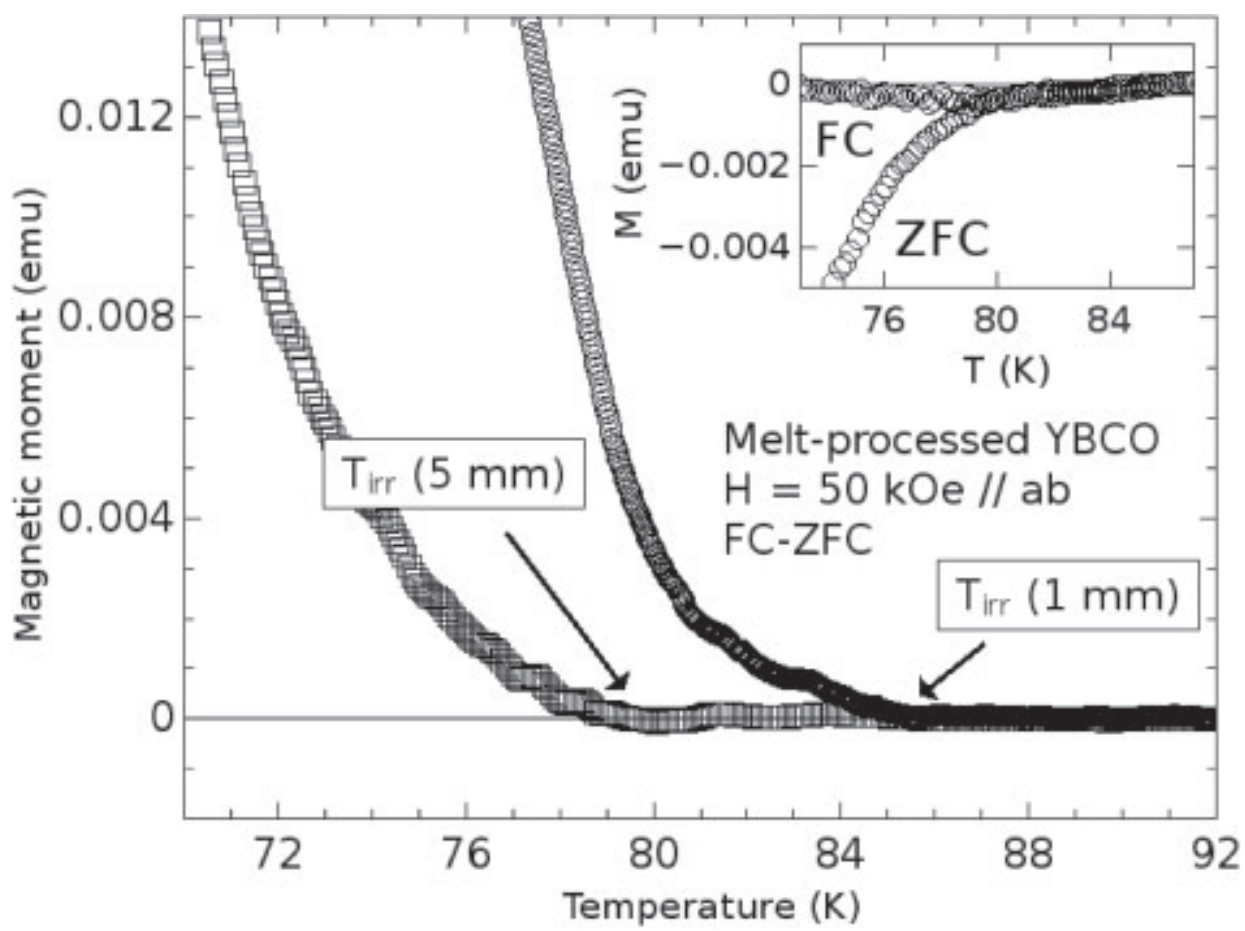

Fig. 3. Difference between FC and ZFC magnetic moments for a magnetic field of $50 \mathrm{kOe}$ applied along the ab plane of the melt-processed sample, exemplifying our criterion to determinate the irreversibility limit $\operatorname{Tirr}(\mathrm{H})$. Accordingly the irreversibility limit is the point where the first points in the FC-ZFC curve lift up beyond the statistical distribution of the points. The arrow indicates the irreversibility limit according to this criterion. The measurementes were performed with amplitudes of the sample transport of 1 and $5 \mathrm{~mm}$. The inset shows the ZFC and FC curves obtained with an amplitude of $5 \mathrm{~mm}$.

Initially our zero resistance and magnetic irreversibility data showed an inconsistency, because the TcO line fell above the Tirr $(\mathrm{H})$ line. Such a result clearly contradicts known properties of superconductivity and could only be attributed to a drawback of the measuring system. After checking several hypotheses, we questioned the homogeneity of the applied magnetic field. While in the transport measurements the sample rests fixed in the sample space, in the magnetic measurements it is moved and cycles in the slightly inhomogeneous field. Such field cycling is well known to induce relaxation toward the equilibrium,16) especially in the ZFC branch. In order to verify the effects of the field cycling in the inhomogeneous magnetic field, we have performed additional ZFC and FC measurements as a function of the amplitude of the sample transport. Figure 4 is a representative result showing how the magnetic irreversibility temperature limit, in a given applied field, varies as a function of the amplitude in the sample transport, $50 \mathrm{kOe}$ in the case of this figure.

Figure 4 lets clear that in order to compare the magnetic irreversibility limit of a superconducting sample with the zero resistance limit, all the experimental circumstances 
must be exactly the same, including the parameters of sample transport. Hence, in order to compare magnetic irreversibility limits with the zero resistance limits in electric transport, in which the sample does not move at all, it is necessary to obtain magnetic irreversibility limits for zero amplitude in the sample transport. The flagrant contradiction of our previous magnetic irreversibility data and the zero resistance data with known properties of superconductors has made us to stumble on this problem. In our view, many published magnetic irreversibility curves of spin-glass systems and superconductors are considerably flawed because of relaxation, due to the inhomogeneous applied field. The importance of the experimental procedure to determinate the Tirr was discussed in details in a previous work by Suenaga et al.17)

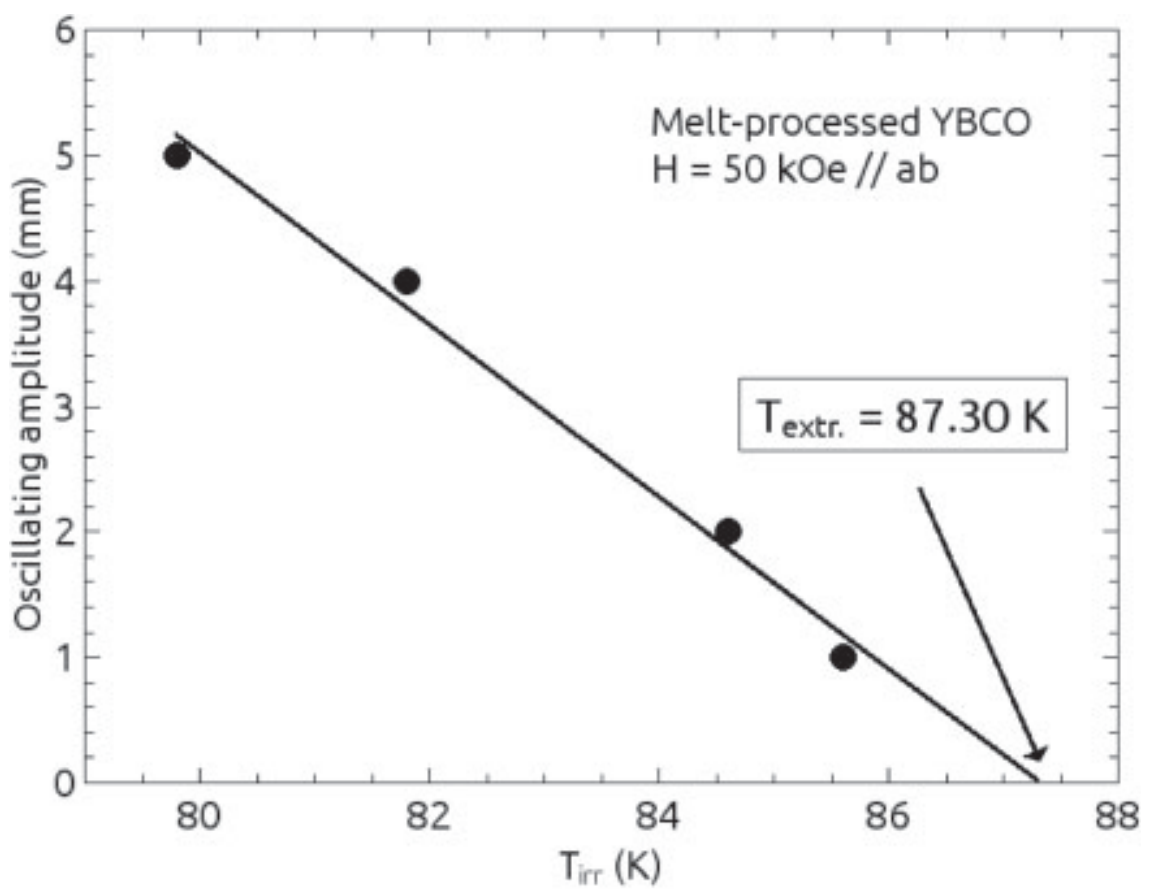

Fig. 4. The magnetic irreversibility limit Tirr as a function of the amplitude of the sample transport from 5 to $1 \mathrm{~mm}$, obtained with the VSM under $50 \mathrm{kOe}$, applied along the ab plane of our sample. Textr is the irreversibility limit extrapolated to zero amplitude.

\section{Results and discussion}

The magnetic irreversibility limit line $\operatorname{Tirr}(\mathrm{H})$ of our samples was determined for applied magnetic fields ranging from 0.01 to $50 \mathrm{kOe}$. Figure 5 displays the irreversibility lines of our melt-processed sample for magnetic fields $\mathrm{H}$ applied along the c-axis ( $\mathrm{H} \| \mathrm{c}$, red dots) and along the ab plane ( $\mathrm{H} \| \mathrm{ab}$, blue dots) "before" and "after" the corrections for sample transport amplitude. The continuous lines (black) through the Tirr $(\mathrm{H})$ data are fittings with the 
usual equation describing the profile of magnetic irreversibility as a function of the applied magnetic field related with the giant flux creep (gfc) model:18)

$$
\text { HirrðTP } 1 / 4 \text { H0ð1_tP_ð_1/43 3=2P: む1P }
$$

In Eq. (1) $t=\operatorname{Tirr}(H)=\operatorname{Tirr}(0)$ is the reduced temperature, $\mathrm{HO}$ is the value of the irreversibility field at zero temperature and $\operatorname{Tirr}(0)$ is the irreversibility temperature at zero applied field. The fitting parameters in Table I (especially the exponent $\alpha$ ) show that magnetic irreversibility data are well fitted with Eq. (1).

Table I. Fitting parameters with Eq. (1) for our melt-processed YBa2Cu3O7- $\delta$ sample with 30 wt\% of Y211 phase inclusions.

$\begin{array}{lllll}\text { Field direction } & \text { Fit } & \alpha & \text { HO (kOe) } & \text { Tirr(0) (K) } \\ (\mathrm{H} \| \mathrm{ab}) & \text { gfc } & 1.50 \pm 0.08 & 6075.92 & 91.02 \\ (\mathrm{H} \| \mathrm{c}) & \text { gfc } & 1.60 \pm 0.04 & 991.81 & 91.01\end{array}$

The anisotropy of the irreversibility lines for $\mathrm{H} \| \mathrm{c}$ and for $\mathrm{H} \|$ ab is large, of the order of that observed in $\mathrm{YBa} 2 \mathrm{Cu} 3 \mathrm{O} 7-\delta$ single crystals. This shows that the c-axis of the crystallites in our samples are well aligned. These irreversibility lines do not exhibit the de Almeida-Thouless14) and the Gabay- Toulouse15) regimes in the low field region, which are the well known signature of superconducting granularity.19) This attests to the good crystallite alignment as well as the strong coupling of the superconducting order parameter between the superconducting grains.

Figure 5 also displays the zero resistance data as a function of applied magnetic field for all the five possible configurations of applied magnetic fields and measuring currents. The continuous lines through these data are only a guide to the eye. 


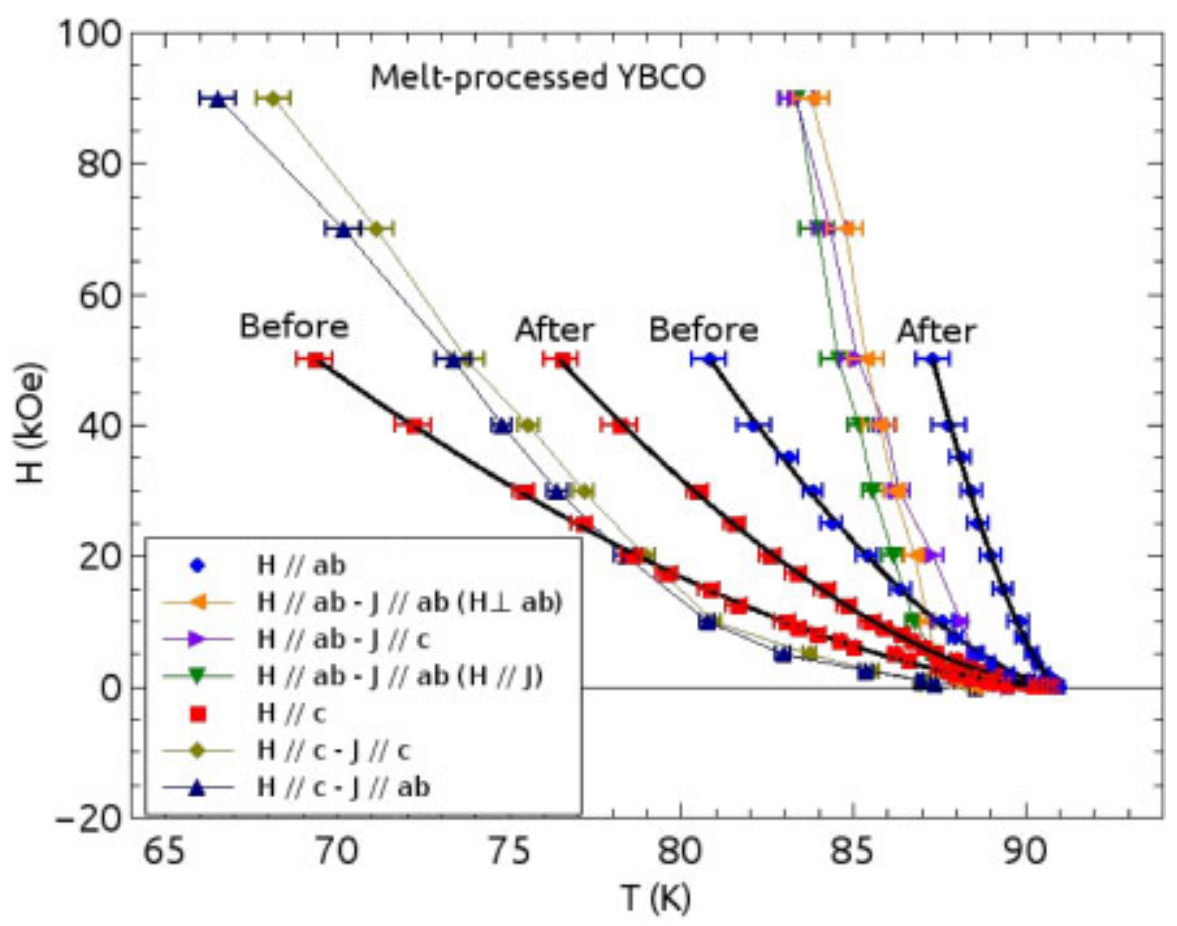

Fig. 5. (Color online) The magnetic irreversibility lines $\operatorname{Tir}(\mathrm{H})$ for $\mathrm{H} \|$ ab and for $\mathrm{H} \| \mathrm{c}$ "before" and "after" correction for sample transport amplitude. The figure also displays the zero resistance lines $\mathrm{TCO}(\mathrm{H})$ for $\mathrm{H} \|$ ab and $\mathrm{H} \| \mathrm{c}$ for all the possible field-current configurations, as listed in the inset of the figure. The lines through the $\operatorname{Tirr}(H)$ data are fittings with Eq. (1) and the fine lines through the $\mathrm{TCO}(\mathrm{H})$ data are only guides to the eye.

On confronting the previous $\operatorname{Tirr}(\mathrm{H})$ lines with the $\mathrm{TCO}(\mathrm{H})$ lines, it was observed that the zero resistance data fell above the irreversibility line, which immediately evidenced problems with the used experimental method. We then used hysteresis cycles to determine the magnetic irreversibility, but the problems went on. After testing various hypotheses, we discovered that the magnetic irreversibility determined from dc-magnetization measurements depends strongly on the amplitude of the sample transport. The obvious reason is that the magnetic field within the sample space is not sufficiently homogeneous. It became clear that in order to correlate magnetic irreversibility with zero resistance, in which the sample does not move within the applied magnetic field, $\operatorname{Tirr}(\mathrm{H})$ must be obtained for zero amplitude in the sample transport. Obviously this is possible only by measuring for several amplitudes and extrapolating to zero amplitude. The simple conclusion is that in order to get a meaningful correlation between magnetic irreversibility lines, $\operatorname{Tir}(H)$, and zero resistance lines, $\operatorname{TcO}(H)$, both measuring data must be obtained under identical measuring conditions, there including the sample transport. 
After correcting the $\operatorname{Tir}(\mathrm{H})$ curves for zero amplitude of the sample transport, it is observed (see Fig. 5) that all the zero resistance lines, as a function of applied magnetic field, $\mathrm{TcO}(\mathrm{H})$ fall nicely underneath the $\operatorname{Tirr}(\mathrm{H})$ lines for all the field and measuring current configurations. This shows that electric resistance below the superconducting transition temperature effectively is caused by excitation of magnetic flux dynamics and that flux pinning at some temperature below the irreversibility temperature, prevents the measuring current from dissipating its energy by inducing flux dynamics. For fields applied along the c-axis the zero resistance lines for the two possible field-current configurations fall closely together and, within the error bars, coincide with each other. However, for low applied fields along the ab plane, the $\mathrm{TcO}(\mathrm{H})$ points lie significantly below the $\operatorname{Tirr}(\mathrm{H})$ line. We attribute this to under-profit of the pinning centers by the low fluxon density.

\section{Conclusions}

Our magnetic irreversibility measurements gave, by the first time, an explicit demonstration of the experimental problem with the standard method for measuring the magnetic irreversibility by DC magnetization, using a VSM. This method consists in measuring the magnetization as a function of temperature, $\mathrm{M}(\mathrm{T})$, in constant applied field while slowly warming the sample after ZFC and subsequently measuring $M(T)$ while cooling it back in the same field. The origin of the problem is the magnetic relaxation, induced by field cycling the sample by transport in the weakly inhomogeneous applied magnetic field of commercial magnetometers. We also have found analogous discrepancies in correlating the magnetic irreversibility line, measured with a MPMS magnetometer from Quantum Design, with zero resistance data on the same sample. We believe that such problems with the magnetic irreversibility limit are common to all DC magnetometers. The origin is the insufficiently homogeneous magnetic field. Such deviation causes very significant displacement of the magnetic irreversibility limit. In the case of our meltprocessed $\mathrm{Y} 123$ sample, the displacement of Tirr $(\mathrm{H})$ reached $7 \mathrm{~K}$ at $50 \mathrm{kOe}$. However, in samples with strong relaxation, such deviations too are not negligible in DC-magnetization curves in general. In order to get consistency between the $\operatorname{Tirr}(\mathrm{H})$ and zero resistance $\operatorname{TcO}(\mathrm{H})$ lines, we measured the magnetic irreversibility as a function of the amplitude of the sample transport and extrapolated it to zero amplitude. Fortunately the displacement of the magnetic irreversibility limit changes linearly with the sample transport amplitude so that the extrapolation was simple to do. This, however, took a very important expenditure of measuring time.

\section{Acknowledgments}


This work was supported under the Brazilian grant MCTI= CNPq Universal 14=2012 (contract number $477506=2012-7)$. The experimental work at the HLD was supported by Euromagnet II (contract number 228043).

\section{References}

1) V. N. Vieira and J. Schaf, Phys. Rev. B 65, 144531 (2002).

2) V. N. Vieira, P. Pureur, and J. Schaf, Phys. Rev. B 66, 224506 (2002).

3) E. Dagotto, Science 309, 257 (2005).

4) J. Rosenblatt, P. Peyral, A. Raboutou, and C. Lebeau, Physica B 152, 95 (1988).

5) J. Figueras, T. Puig, and X. Obradors, Phys. Rev. B 67, 014503 (2003).

6) T. Puig, F. Galante, E. M. Gonzales, J. L. Vicent, B. Martínez, and X. Obradors, Phys. Rev. B 60, 13099 (1999).

7) J. Figueras, T. Puig, X. Obradors, W. K. Kwok, L. Paulius, G. W. Crabtree, and G. Deutscher, Nat. Phys. 2, 402 (2006).

8) X. Granados, S. Piñol, B. Martínez, F. Galante, F. Sandiumenge, J.Fontcuberta, and X. Obradors, Cryogenics 34, 833 (1994).

9) F. Sandiumenge, S. Piñol, X. Obradors, E. Snoeck, and C. Roucau, Phys. Rev. B 50, 7032 (1994).

10) S. Piñol, V. Gomis, B. Martínez, A. Labarta, J. Fontcuberta, and X. Obradors, J. Alloys Compd. 195, 11 (1993).

11) S. Piñol, F. Sandiumenge, B. Martínez, V. Gomis, J. Fontcuberta, X. Obradors, E. Snoeck, and C. Roucau, Appl. Phys. Lett. 65, 1448 (1994).

12) X. Obradors, R. Yu, F. Sandiumenge, B. Martínez, N. Vilalta, V. Gomis, T. Puig, and S. Piol, Supercond. Sci. Technol. 10, 884 (1997).

13) S. Sanfilippo, A. Sulpice, O. Laborde, D. Bourgault, Th. Fournier, and R. Tournier, Phys. Rev. B 58, 15189 (1998).

14) J. R. L. de Almeida and D. J. Thouless, J. Phys. A 11, 983 (1978).

15) M. Gabay and G. Toulouse, Phys. Rev. Lett. 47, 201 (1981). 
16) Y. Yeshurun, A. P. Malozemoff, and A. Shaulov, Rev. Mod. Phys. 68, 911 (1996).

17) M. Suenaga, D. O. Welch, and R. Budhani, Supercond. Sci. Technol. 5, S1 (1992).

18) Y. Yeshurun and A. P. Malozemoff, Phys. Rev. Lett. 60, 2202 (1988).

19) F. T. Dias, V. N. Vieira, P. Rodrigues, Jr., X. Obradors, P. Pureur, and J. Schaf, Phys. Rev. B 77,134503 (2008). 